\title{
Pediatric Nocardial Brain Abscesses in Acquired Immunodeficiency Syndrome
}

\author{
Chotey NA ${ }^{1}$, Ramdial PK $^{1 *}$, Miles $E^{1}$, Nargan $K^{2}$ and Mubaiwa $L^{3}$ \\ ${ }^{1}$ Department of Anatomical Pathology, National Health Laboratory Service \& University of KwaZulu-Natal, Durban, South Africa \\ ${ }^{2}$ KwaZulu-Natal Research Institute for Tuberculosis and HIV, Durban, KwaZulu-Natal, South Africa
}

${ }^{3}$ Paediatrics and Child Health, Nelson R Mandela School of Medicine, University of KwaZulu-Natal, Durban, South Africa

\begin{abstract}
Nocardiosis is relatively uncommon in children and adults with acquired immunodeficiency syndrome (AIDS), despite the profound associated cellular immunodeficiency. Acquired most often by inhalation and less commonly by percutaneous inoculation, subsequent hematogenous dissemination may lead to infection of almost any organ, with a particular predilection for the central nervous system. Nocardial brain abscesses are rare. To the best of our knowledge, pediatric Nocardial brain abscesses have not been documented in Human Immunodeficiency Virus (HIV)-infected children in the English-language literature, to date. In reporting two Nocardial brain abscesses in a 9 year old AIDS patient with intermittent seizures, we highlight the difficulty associated with the ante-mortem diagnosis of Nocardial brain abscesses, and the need for cognizance of rare entities occurring in HIV-infected children. Furthermore, we emphasize the pivotal role of the autopsy in finalizing the nature of the cerebral pathology, the cause of the seizures albeit post-mortem, a cause of death and in providing a platform for continued learning in the AIDS era.
\end{abstract}

Keywords: Abscess; AIDS; HIV; Brain; Nocardial; Pediatric; Autopsy

\section{Introduction}

Nocardiosis is a localized or systemic infection caused by Grampositive, weakly acid fast, filamentous, strictly aerobic actinomycetes belonging to the genus Nocardia in the order Actinomycetales [1]. It occurs more commonly in patients with impaired immunity, especially males in the $3^{\text {rd }}$ and $4^{\text {th }}$ decades [1-3]. Nocardiosis is relatively uncommon in children and adults with acquired immunodeficiency syndrome (AIDS), despite the profound associated cellular immunodeficiency [2-5]. Acquired most often by inhalation and less commonly by percutaneous inoculation, subsequent hematogenous dissemination may lead to infection of almost any organ, with a particular predilection for the central nervous system (CNS), the latter being involved in 20$40 \%$ of patients $[2,5]$. Notwithstanding the CNS spectrum, Nocardial brain abscesses are rare, representing only $2 \%$ of all cerebral abscesses [6]. To the best of our knowledge, pediatric Nocardial brain abscesses have not been documented in Human Immunodeficiency Virus (HIV)infected children in the English-language literature, to date.

In reporting two Nocardial brain abscesses in an HIV-infected child, we highlight the need for cognizance of rare entities occurring in HIV-infected children. Furthermore, we emphasize the pivotal role of the autopsy in finalizing a cause of death and in providing a platform for continued learning in the AIDS era.

\section{Case Report}

\section{Clinical features}

A 9 year old Black African male was admitted to a peripheral hospital with a 6 month history of intermittent seizures that involved the right side of the face without loss of consciousness. He was initiated on anti-convulsant therapy. The patient was discharged but required re-admission because of poor seizure control. At this stage, the patient was also found to be HIV-seropositive with a CD4 count of 105 cells/ $\mu \mathrm{L}$. A brain computed tomography (CT) scan was performed at the peripheral hospital. It revealed ring-enhancing lesions that involved the right basal ganglia and thalamus and left parietal regions. There was partial aqueductal obstruction, raised intracranial pressure and left midline shift. The radiological considerations were tuberculosis and toxoplasmosis. He was initiated on highly active anti-retroviral therapy (HAART) (efavirenz, stavudine, lamivudine), morphine, paracetamol, metronidazole and fluconazole and was referred urgently to the academic unit for definitive management. A contrastenhanced magnetic resonance imaging (MRI) scan was undertaken at the academic unit; it confirmed the CT scan findings (Figure 1). The patient was booked for immediate neurosurgical intervention at the academic center but developed respiratory distress and demised before further investigations and management.

The clinical information was incomplete in respect of the clinical investigations undertaken at the peripheral hospital. The referral consultation letter confirmed that a lumbar puncture had been performed but the cerebrospinal fluid did not reveal any gross or microscopic abnormalities; however, the detailed cerebrospinal fluid results were not provided. The patient's serological biochemical profiles were also not included therein. The clinical records did not contain information on the parental HIV status or the history of the patient's HIV disease. Attempts to contact the parents after the autopsy failed.

\section{Autopsy findings}

Gross features: The patient's height and weight were $115.4 \mathrm{~cm}$ and $15.3 \mathrm{~kg}$, respectively. Lymphadenopathy or mucocutaneous lesions were not identified on external examination. The lungs demonstrated

*Corresponding author: PK Ramdial, Department of Anatomical Pathology, Leve 3, Laboratory Building, Inkosi Albert Luthuli Central Hospital, 800 Vusi Mzimela Road, Mayville, 4058, KwaZulu-Natal, South Africa; Tel: +27 (0)31 2402693; Fax: +27 (0)31 2402610; E-mail: ramdial@ukzn.ac.za, ramdialpk@gmail.com

Received September 04, 2016; Accepted October 12, 2016; Published October 19, 2016

Citation: Chotey NA, Ramdial PK, Miles E, Nargan K, Mubaiwa L (2016) Pediatric Nocardial Brain Abscesses in Acquired Immunodeficiency Syndrome. J AIDS Clin Res 7: 628. doi: 10.4172/2155-6113.1000628

Copyright: (c) 2016 Chotey NA, et al. This is an open-access article distributed under the terms of the Creative Commons Attribution License, which permits unrestricted use, distribution, and reproduction in any medium, provided the original author and source are credited. 
Citation: Chotey NA, Ramdial PK, Miles E, Nargan K, Mubaiwa L (2016) Pediatric Nocardial Brain Abscesses in Acquired Immunodeficiency Syndrome. J AIDS Clin Res 7: 628. doi: 10.4172/2155-6113.1000628

congestion and mild firmness without evidence of consolidation, friability, cavitation or abscesses. The brain weighed $1400 \mathrm{~g}$. It was swollen and demonstrated features of raised intracranial pressure, including herniation of the left uncus and parahippocampal gyrus. Meningitis was not evident; culture of a meningeal swab was negative. Apart from non-specific terminal autopsy findings, the other organs did not demonstrate any specific abnormalities.

The brain was sectioned following two weeks formalin fixation. The gross findings were congruent with that noted on the CT and MRI scans (Figure 1). Two abscesses were noted in the region of the right basal ganglia and thalamus and left parietal region (Figure 2). There was associated asymmetric cerebral swelling, displacement of the midline structures to the right and distortion of the lateral ventricles and aqueduct.

Microscopic features: Sections of the brain abscesses confirmed non-specific chronic suppurative inflammation and a surrounding gliomesenchymal reaction (Figure 3A). The Gomori Grocott methenamine silver (GGMS) (Figure 3B) and modified Gram (Brown Brenn Hopps) (Figure 4) stains revealed approximately $1 \mu \mathrm{m}$ slender, filamentous beaded Gram-positive bacteria. These demonstrated frequent right angled branching. The Ziehl Neelsen and periodic acid Schiff (PAS) stains did not demonstrate concomitant mycobacteria or fungi, respectively. A direct smear of the abscess contents demonstrated poorly preserved, fragmented, beaded, filamentous acid fast organisms on Kinyoun staining (Figure 4). The branching, filamentous morphology of the organisms and associated Gram and Kinyoun positive staining were typical of Nocardial organisms. Autopsy budgetary constraints prohibited molecular investigation of the exact Nocardial species by polymerase chain reaction and sequencing.

Sections of the lung demonstrated a polymorphous infiltrate of lymphocytes, plasma cells and histiocytes in the interstitium and interlobular septa. Nodular lymphoid aggregates with focal small germinal centers were noted. The lymphocytic component displayed a mixed $\mathrm{B}$ and $\mathrm{T}$ immunohistochemical profile, with a predominance

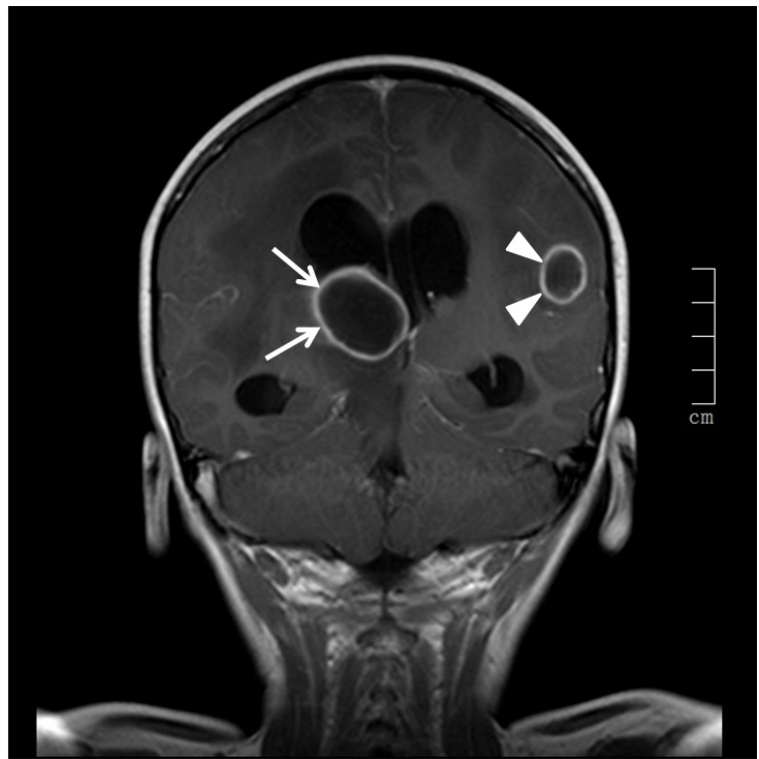

Figure 1: Coronal post-contrast magnetic resonance imaging scan of abscesses in the right basal ganglia and thalamus (arrows) and the left parietal (arrowheads) regions.

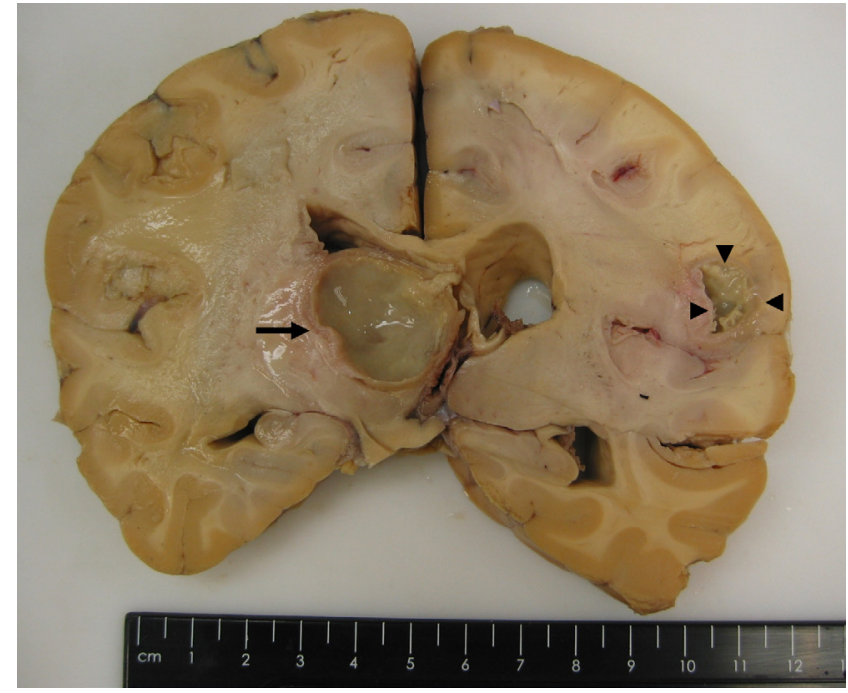

Figure 2: Abscesses in the right basal ganglia and thalamus (arrow) and the left parietal (arrowheads) regions.

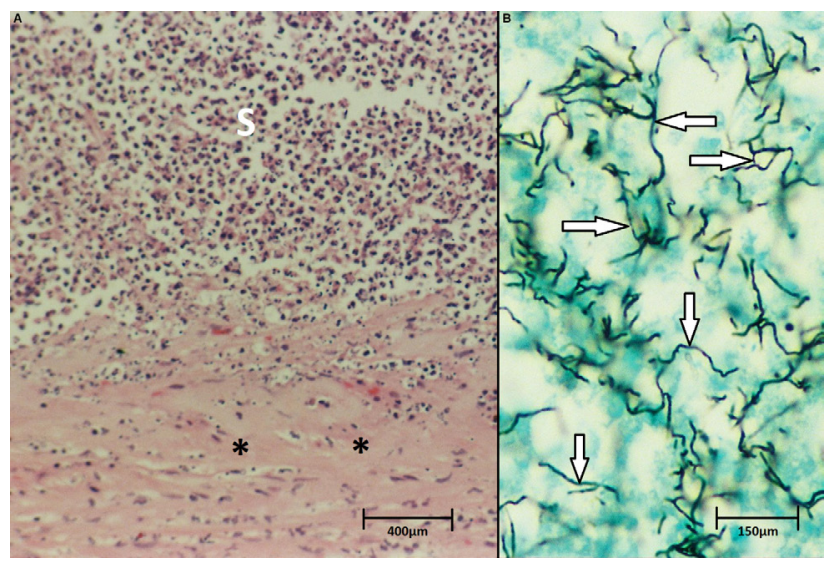

Figure 3: A. Abscess wall with central suppuration (S) and peripheral granulation tissue with fibrosis (asterisks) [Hematoxylin \& eosin, 240X]. B. Branching, filamentous organisms (arrows) [Gomori Grocott methenamine silver, 480X].

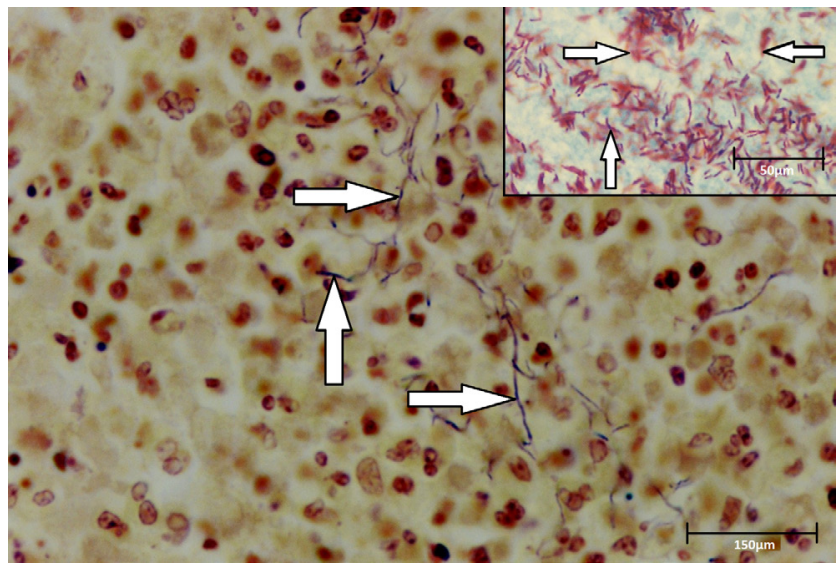

Figure 4: Filamentous, Gram-positive, branching organisms [Brown Brenn Hopps, 1200X]. Inset: Direct smear of abscess contents with fragmenting, filamentous, weakly acid fast organisms (arrows) [Kinyoun, 1200X]. 
of the latter. Immunohistochemical staining of the T-lymphocytic component confirmed a mixed $\mathrm{CD}^{+} / \mathrm{CD}^{+}$infiltrate with a predominant $\mathrm{CD}^{+}$immunophenotype. The features were in keeping with lymphocytic interstitial pneumonia. Granulomas or abscesses were not identified. Ziehl Neelsen, PAS and GGMS stains did not reveal Nocardial or other infective agents. Sections of the other organs did not reveal any specific pathology.

The final autopsy diagnosis was that of AIDS with Nocardial brain abscesses, associated raised intracranial pressure, lymphoid interstitial pneumonia and respiratory failure.

\section{Discussion}

Since the first documentation of human nocardiosis more than a century ago, Nocardial infections have been increasingly recognized as a serious, primarily opportunistic, infection. This has been ascribed mainly to an increasing population of immunocompromized hosts, wider use of immunosuppressive therapy, improved methods for detection and identification of Nocardia species, and a heightened index of suspicion among physicians [6,7]. Thirteen medically important species are contained within the genus Nocardia. Of these, $N$. asteroides, $N$. farcinica, $N$. nova and $N$. abscessus are responsible for the majority of invasive infections, but $N$. asteroides is identified most commonly [8]. The anti-Nocardial host defense mechanisms include initial bacterial inhibition by neutrophils and macrophages and subsequent $\mathrm{T}$-cell mediated bacterial destruction $[1,3,8]$. Nocardial virulence factors include the catalase and superoxide dismutase enzymes that enable Nocardial inactivation of neutrophil-generated reactive oxygen species $[8,9]$. In addition, 'cord factor', a Nocardial cell wall glycolipid, obstructs macrophage activation and associated phagocytosis and phagolysosome formation $[8,10]$. Approximately one third of patients afflicted by nocardiosis are immunocompetent [8].

The incidence of primary cerebral nocardiosis in the absence of extraneural involvement ranges from 5 to $33 \%$, while secondary CNS involvement, caused by a spectrum of underlying diseases, occurs in $15-44 \%$ of patients $[8,11,12]$. The clinical presentations of patients with CNS nocardiosis are non-specific but headache, fever, altered mental state, seizures, visual changes and nausea and vomiting have been reported in $>20 \%$ of cases globally [8]. The range of Nocardial CNS pathology includes spinal cord lesions, diffuse cerebral infiltration, leptomeningitis, granulomas, ventriculitis and cerebral abscess formation [6,11]. While CNS Nocardial infection manifests overwhelmingly as a solitary abscess in a predominantly supratentorial location, multiple brain abscesses are reported in 38\% of cerebral nocardiosis [13]. Apart from HIV infection and AIDS, Nocardial brain abscesses, in particular, have been associated with a range of local and systemic diseases (Table 1) [8,14-16]. Nocardial brain abscesses are caused by $N$. asteroides, $N$. farcinica, $N$. nova, $N$. paucivorans, $N$. brasiliensis, $N$. cyriacigeorgical, $N$. transvalensis and N. otitidiscaviarum [8,14,17-19]. Even in the presence of systemic nocardiosis, diagnostic aspiration of brain abscesses is recommended to evaluate co-infections by multiple organisms [14]. Disseminated nocardiosis with cerebral involvement has a very poor prognosis because the diagnosis of cerebral disease is seldom confirmed antemortem [20]. Leptomeningitis is an uncommon event. The cerebrospinal fluid is often normal even with an established intracerebral abscess [5]; this was the situation in the index patient. Although Nocardial brain abscesses occur mainly in immunocompromized patients, similar to other bacterial brain abscesses, no risk factors for infection are identified in $>50 \%$ of patients with Nocardial abscesses [3].

\begin{tabular}{|c|c|}
\hline INFLAMMATORY CONDITIONS & ORG \\
\hline 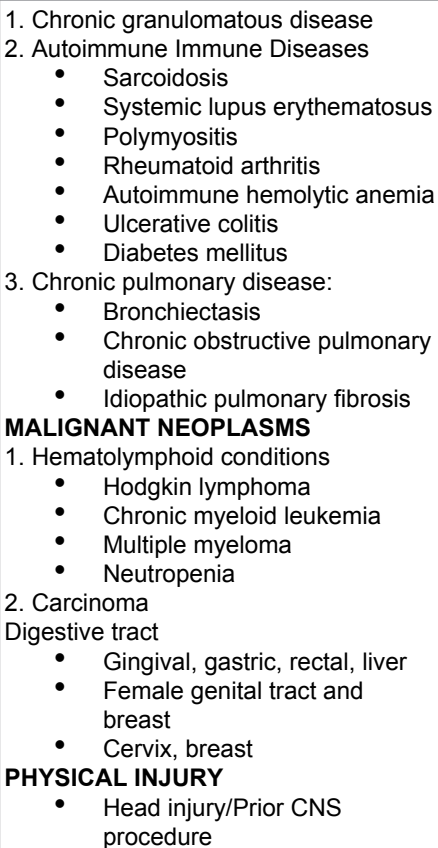 & 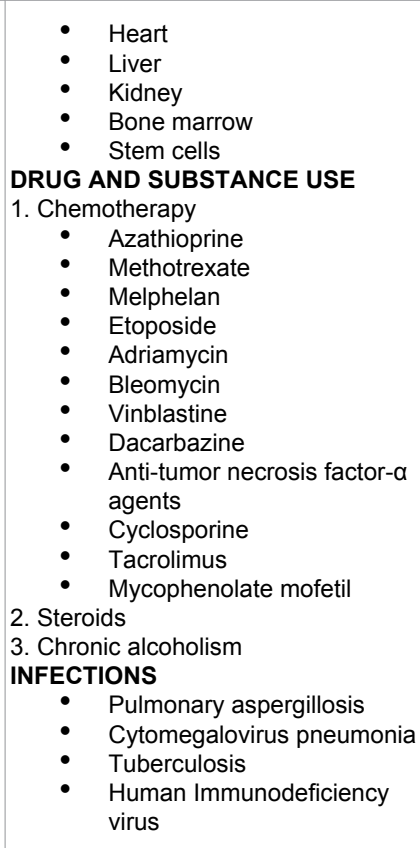 \\
\hline
\end{tabular}

CNS: Central Nervous System

Table 1: Conditions/diseases associated with Nocardial brain abscesses [8,14-16]

HIV infection as a predisposing factor in the causation of nocardiosis was recognized in the 1980s, but HIV and Nocardial coinfection remains an uncommon occurrence, with rates of $0.2-1.8 \%$ being reported $[7,21]$. Pulmonary nocardiosis with secondary cerebral disease is reported as the common mode of presentation in AIDS patients, but diverse disease presentations such as joint and bone infection, paraspinal and renal abscesses and adrenal insufficiency have expanded the clinical spectrum in the AIDS era [4].

The male predominance, range of organ involvement, association with an underlying host defect and the $40 \%$ mortality rate of nocardiosis in children, has paralleled the spectrum of disease reported in adults [22,23]. Pediatric nocardiosis, however, is a rarity in the global literature, even from developing countries ravaged by the AIDS epidemic; to date, cerebral Nocardial brain abscesses in pediatric AIDS patients are undocumented. Whether this is a true low incidence or whether the low incidence is an under-recognition or under-reporting phenomenon, especially since nocardiosis is not a Centers of Disease Control AIDSdefining disease [7], remains conjectural. Ring-enhancing lesions in HIV-positive patients are presumed to be of T. gondii origin; patients are commenced on sulfonamide therapy, to which Nocardia may also be adequately sensitive. Also, many HIV positive patients receive trimethoprim sulfamethoxazole prophylaxis against Pneumocystis and other opportunistic infections. Such prophylaxis may prevent or treat Nocardia infection curatively $[3,14]$. Our patient had been on prophylactic anti-fungal and anti-bacterial agents for only 2 days prior to death. The Nocardial nature of the space occupying masses was unrecognized clinically; the lumbar puncture did not confirm meningitis and there were no extraneural clues of nocardiosis. In the index patient, AIDS and the attendant immunocompromize are the posited risk factors for cerebral nocardiosis, but it is arguable whether the brain abscesses were primary or secondary. Despite the absence of extracerebral systemic nocardiosis at autopsy, it is not possible to exclude that the use of antibiotics for other undeclared childhood 
illnesses, could have unintentionally treated undetected systemic disease.

The mortality rate of nocardiosis is $75-90 \%$ [5]. Factors predictive of a better outcome include early diagnosis, absence of underlying disease, administration of appropriate chemotherapy and restricted disease $[5,8,14]$. Mortality is also higher in those undergoing stereotactic aspiration (50\%) than in those undergoing craniotomy (24\%) or in those managed non-operatively [5]. There was limitation of the disease to the brain terminally, but the intracerebral multifocality, presence of underlying AIDS, lack of a timely diagnosis and timely appropriate medication provided a predictable permutation for poor prognosis. Had it not been for the autopsy, confirmation of nocardiosis would not have occurred.

Although the autopsy has been described as the "ultimate medical consultation" and the "ultimate audit", the $20^{\text {th }}$ and $21^{\text {st }}$ centuries have experienced a marked global decline in autopsy rates. While many reasons for the decline exist, perhaps the most influential is the advances in medical technology that provide ante-mortem diagnosis, rendering physicians less uncertain of a cause of death. However discrepancies of approximately $10 \%$ between clinical and autopsy diagnoses are recorded and the autopsy is critical as a monitor of clinical investigative techniques [24]. Furthermore, autopsies are a critical continuing professional developmental tool, especially teaching and learning on new, emerging diseases and new presentations of old diseases. The learning on disease profiles in the HIV and AIDS era touches on both scenarios. While Nocardia species, with their characteristic morphological and staining profile, facilitated a definitive diagnosis of Nocardial brain abscesses, albeit post-mortem, the exact species identification remains unknown, because of the lack of positive antemortem Nocardial culture, and unsuitability of the post-mortem, formalin-fixed abscess contents for microbiological culture. Clinicians must inform the laboratory when the clinical suspicion of Nocardial infection is strong as prolonged culture may be necessary because some Nocardia species are slow growers [25]. Nocardial species identification from formalin-fixed paraffin-embedded tissue, although not undertaken because of autopsy budgetary constraints in the index patient, is important, moreso in ante-mortem biopsies, because not all Nocardia species are pathogenic and those that are, have known, markedly different susceptibility and resistance patterns [26].

In adding pediatric AIDS-associated Nocardial brain abscesses to the world literature, we emphasize the almost perfunctory consideration of Nocardia species as a cause for ring-enhancing lesions on imaging studies in children, especially in those who are HIV-infected, even when cerebrospinal fluid testing does not confirm meningitis.

\section{Acknowledgement}

Ms. M Moodley for manuscript typing. The NHLS management for encouragement and support of research and training pillars. This case report followed the guidelines of the Biomedical Research Committee of the University of KwaZulu-Natal.

\section{References}

1. Grange JM (2002) Actinomyces, Nocardia and tropheryma, Actinomycosis; nocardiosis, Whipple's disease. Medical Microbiology, Churchill Livingstone, Edinburgh, pp: 221-224.

2. Javaly K, Horowitz HW, Wormser GP (1992) Nocardiosis in patients with human immunodeficiency virus infection. Report of 2 cases and review of the literature. Medicine (Baltimore) 71: 128-138.

3. Mamelak AN, Obana WG, Flaherty JF, Rosenblum ML (1994) Nocardial brain abscess: Treatment strategies and factors influencing outcome. Neurosurgery 35: 622-631.
4. Jones N, Khoosal M, Louw M, Karstaedt A (2000) Nocardial infection as a complication of HIV in South Africa. J Infect 41: 232-239.

5. Hall WA, Martinez AJ, Dummer JS, Lunsford LD (1987) Nocardial brain abscess: Diagnostic and therapeutic use of stereotactic aspiration. Surg Neurol 28: 114-118.

6. Kennedy KJ, Chung KH, Bowden FJ, Mews PJ, Pik JH, et al. (2007) A cluster of Nocardial brain abscesses. Surg Neurol 68: 43-49.

7. Kim J, Minamoto GY, Grieco MH (1991) Nocardial infection as a complication of AIDS: Report of six cases and review. Rev Infect Dis 13: 624-629.

8. Anagnostou T, Arvanitis M, Kourkoumpetis TK, Desalermos A, Carneiro HA, et al. (2014) Nocardiosis of the central nervous system: Experience from a general hospital and review of 84 cases from the literature. Medicine (Baltimore) 93: 19-32.

9. Beaman BL, Black CM, Doughty F, Beaman L (1985) Role of superoxide dismutase and catalase as determinants of pathogenicity of Nocardia asteroides: Importance in resistance to microbicidal activities of human polymorphonuclear neutrophils. Infect Immun 47: 135-141.

10. Beaman BL, Beaman L (1994) Nocardia species: Host-parasite relationships. Clin Microbiol Rev 7: 213-264.

11. Fleetwood IG, Embil JM, Ross IB (2000) Nocardia asteroides cerebral abscess in immunocompetent hosts: Report of three cases and review of surgical recommendations. Surg Neurol 53: 605-610.

12. Jain N, Duggal L, Rohtagi A, Sharma A (2005) Nocardiosis in AIDS - An unusual presentation. J Assoc Physicians India 53: 1052.

13. Mogilner A, Jallo GI, Zagzag D, Kelly PJ (1998) Nocardia abscess of the choroid plexus: Clinical and pathological case report. Neurosurgery 43: 949-952.

14. Bross JE, Gordon G (1991) Nocardial meningitis: Case reports and review. Rev Infect Dis 13: 160-165.

15. Alavi Darazam I, Shamaei M, Mobarhan M, Ghasemi S, Tabarsi P, et al (2013) Nocardiosis: Risk factors, clinical characteristics and outcome. Iran Red Crescent Med J 15: 436-439.

16. Chow E, Moore T, Deville J, Nielsen K (2005) Nocardia asteroides brain abscesses and meningitis in an immunocompromized 10 year old child. Scand J Infect Dis 37: 511-513.

17. Monticelli J, Luzzati R, Maurel C, Rosin C, Romina V, Farina C (2015) Brain abscesses caused by Nocardia paucivorans in a multiple myeloma patient treated with lenalidomide and dexamethasone: A case report and review of literature. Mediterr J Hematol Infect Dis 7: e2015011.

18. Baldawa S, Nayak N, Kukreja S, D'souza D, Diyora B, et al. (2014) Cerebra nocardiosis. Asian J Neurosurg 9: 245.

19. Yorke RF, Rouah E (2003) Nocardiosis with brain abscess due to an unusual species, Nocardia transvalensis. Arch Pathol Lab Med 127: 224-226.

20. Ogg G, Lynn WA, Peters M, Curati W, McLaughlin JE, et al. (1997) Cerebra Nocardia abscesses in a patient with AIDS: Correlation of magnetic resonance and white cell scanning images with neuropathological findings. J Infect 35 : 311-313.

21. Uttamchandani RB, Daikos GL, Reyes RR, Fischl MA, Dickinson GM, et al (1994) Nocardiosis in 30 patients with advanced human immunodeficiency virus infection: Clinical features and outcome. Clin Infect Dis 18: 348-353.

22. Law BJ, Marks MI (1982) Pediatric nocardiosis. Pediatrics 70: 560-565.

23. Marlowe M, Ali-Ahmad D, Cherrick I, Higgins MJ, Kiska DL, et al. (2000) Centra nervous system nocardiosis in an immunocompetent child. Pediatr Infect Dis J 19: 661-662

24. Oluwasola OA, Fawole OI, Otegbayo AJ, Ogun GO, Adebamowo CA, et al. (2009) The autopsy: Knowledge, attitude and perceptions of doctors and relatives of the deceased. Arch Pathol Lab Med 133: 78-82.

25. Wang HK, Sheng WH, Hung CC, Chen YC, Lee MH, et al. (2015) Clinica characteristics, microbiology and outcomes for patients with lung and disseminated nocardiosis in a tertiary hospital. J Formos Med Assoc 114: 742-749.

26. Abreu C, Rocha-Periera N, Sarmento A, Magro F (2015) Nocardia infections among immunomodulated inflammatory bowel disease patients: A review. World J Gastroenterol 21: 6491-6498. 